\title{
Propagation of Uncertainty in Rigid Body Attitude Flows
}

\author{
Taeyoung Lee ${ }^{* \dagger}$, Nalin A. Chaturvedi ${ }^{\dagger}$, Amit Sanyal, Melvin Leok*, and N. Harris McClamroch ${ }^{\dagger}$
}

\begin{abstract}
Motivated by attitude control and attitude estimation problems for a rigid body, computational methods are proposed to propagate uncertainties in the angular velocity and the attitude. Uncertainties in the angular velocity and attitude are described in terms of ellipsoidal sets that are propagated through this nonlinear attitude flow. Computational methods are proposed, one method based on a local linearization of the attitude flow and two methods based on propagation of a small (unscented) sample selected from the initial uncertainty ellipsoid. Each of these computational methods is constructed using a Lie group variational integrator, viewed as a discretization of the attitude dynamics. Computational results are obtained that indicate (1) the strongly nonlinear attitude flow characteristics and (2) the limitations of each of these methods, and indeed any method, in providing effective global bounds on the nonlinear attitude flow.
\end{abstract}

\section{INTRODUCTION}

As an integrable system, the attitude dynamics of the free rigid body are reasonably well understood. However, if there is an attitude dependent potential that influences the rigid body, then the dynamics can be surprisingly complex. In this paper, such attitude dynamics are studied for a rigid body with an inertially fixed pivot acting under the influence of uniform and constant gravity; this model is subsequently referred to as the $3 \mathrm{D}$ pendulum [1].

The objective of this paper is to study the nonlinear attitude flow of the 3D pendulum dynamics by characterizing the propagation of uncertainty for the attitude and angular velocity in a deterministic sense; bounds on the uncertainty are specified and propagated without an assumption on their distribution. This line of research was motivated by our prior work on deterministic attitude estimation [2], [3], which is composed of deterministic uncertainty propagation, measurement update, and filtering procedures.

Uncertainty propagation can also be studied within a probabilistic framework using the Liouville partial differential equation, a special case of the Fokker-Planck equation, but this leads to significant solution difficulties [4]. However, statistical properties of uncertainties are often unavailable or are difficult to obtain in practice. It has been shown that deterministic estimation is more robust to noise distribution [5].

Taeyoung Lee, Nalin A. Chaturvedi, and N. Harris McClamroch, Aerospace Engineering, University of Michigan, Ann Arbor, MI 48109 \{tylee, nalin, nhm $\}$ aumich.edu

Amit Sanyal, Mechanical Engineering, University of Hawaii at Manoa, Honolulu, HI 96822 aksanyal@hawaii.edu

Melvin Leok, Mathematics, Purdue University, West Lafayette, IN 47907 mleok@math. purdue.edu

* This research has been supported in part by NSF under grants DMS0504747 and DMS-0726263.

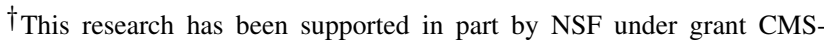
0555797.
In this paper, three computational methods for deterministic attitude uncertainty propagation are numerically compared; one method based on a local linearization of the attitude flow and two methods based on propagation of a small (unscented) sample selected from the initial uncertainty ellipsoid. Our approach in this paper is to make use of geometrically-exact computational tools that respect the Lie group structure of the configuration space for the rigid body attitude dynamics. This approach is based on the recently introduced Lie group variational integrator for the 3D pendulum and on a framework for propagating suitably defined uncertainty ellipsoids [6].

It turns out that these computational tools are useful in some cases, but they have important limitations that arise from the complex attitude dynamics of the 3D pendulum. The results in this paper demonstrate the complex global dynamics that can occur for an uncontrolled 3D pendulum. In this way, the challenges of attitude control and estimation are made clear, at least in the case where global results are desired.

This paper is organized as follows. The 3D pendulum model is described in Section II. Three computation approach for deterministic attitude uncertainty propagation are presented in Section III, and their computational properties are shown in Section IV. The global dynamic characteristics of the 3D pendulum are discussed in Section V.

\section{3D PENDULUM}

A rigid $3 \mathrm{D}$ pendulum is a rigid body supported by a fixed, frictionless pivot, acted on by uniform gravitational forces [1]. The supporting pivot allows the pendulum three rotational degrees of freedom.

Two reference frames are introduced. An inertial reference frame has its origin at the pivot; the first two axes lie in the horizontal plane and the third axis is vertical in the direction of gravity. A reference frame fixed to the pendulum body is also introduced. The origin of this body-fixed frame is also located at the pivot.

The configuration space is the special orthogonal group $S O(3)$,

$$
S O(3)=\left\{R \in \mathbb{R}^{3 \times 3} \mid R^{T} R=I_{3 \times 3}, \operatorname{det} R=1\right\},
$$

where the rotation matrix $R \in S O(3)$ represents the linear transformation from the body-fixed frame to the inertial frame.

The dynamics of the 3D pendulum are given by the Euler rigid body equation that includes the moment due to gravity:

$$
J \dot{\Omega}=J \Omega \times \Omega+m g \rho \times R^{T} e_{3},
$$


where the angular velocity in the body-fixed frame is denoted by $\Omega \in \mathbb{R}^{3}$, the moment of inertia matrix is denoted by $J \in \mathbb{R}^{3 \times 3}$, and the vector $\rho \in \mathbb{R}^{3}$ represents the location of the center of mass in the body-fixed frame. The constants $m$ and $g$ denote the mass of the pendulum and the gravitational acceleration, respectively. The kinematic equation is

$$
\dot{R}=R S(\Omega) \text {. }
$$

For a given vector $a \in \mathbb{R}^{3}$, the $3 \times 3$ skew-symmetric matrix $S(a)$ is defined so that $S(a) b=a \times b$ for all $b \in \mathbb{R}^{3}$.

There are two disjoint equilibria when the direction of gravity in the body-fixed frame is collinear with the vector $\rho$. We define

$$
\begin{aligned}
& H=\left\{(R, \Omega) \in T S O(3) \mid R^{T} e_{3}=\rho /\|\rho\|, \Omega=0\right\}, \\
& I=\left\{(R, \Omega) \in T S O(3) \mid R^{T} e_{3}=-\rho /\|\rho\|, \Omega=0\right\}
\end{aligned}
$$

as hanging equilibria and inverted equilibria, respectively. Here, the tangent bundle of $S O(3)$ is denoted by $T S O(3)$, which can be identified with $S O(3) \times \mathbb{R}^{3}$.

\section{UNCERTAINTY PROPAGATION}

An uncertainty ellipsoid on $T S O(3)$ is defined by

$$
\mathcal{E}(\hat{R}, \hat{\Omega}, P)=\left\{(R, \Omega) \in T S O(3) \mid x^{T} P^{-1} x \leq 1\right\},
$$

where $x=[\zeta ; \delta \Omega] \in \mathbb{R}^{6}$ and $\zeta=\log \left(\hat{R}^{T} R\right), \delta \Omega=\Omega-$ $\hat{\Omega} \in \mathbb{R}^{3}$. The center of the ellipsoid is given by the rotation matrix $\hat{R}$ and the angular velocity $\hat{\Omega}$; the matrix $P \in \mathbb{R}^{6 \times 6}$ is the uncertainty matrix that characterizes the size and the shape of the ellipsoid. In particular, if the initial attitude and angular velocity are known to lie within an initial uncertainty ellipsoid, we seek computational methods to propagate the uncertainty so as to obtain an uncertainty ellipsoid, for the current time, within which the current attitude and angular velocity of the 3D pendulum are expected to lie.

We study three methods to propagate the uncertainty set over the time interval $[0, T]$. All three methods make use of the Lie group variational integrator that is described by the following discrete update equations [6],

$$
\begin{gathered}
h S\left(J \Omega_{k}+\frac{h}{2} M_{k}\right)=F_{k} J_{d}-J_{d} F_{k}^{T}, \\
R_{k+1}=R_{k} F_{k}, \\
J \Omega_{k+1}=F_{k}^{T} J \Omega_{k}+\frac{h}{2} F_{k}^{T} M_{k}+\frac{h}{2} M_{k+1},
\end{gathered}
$$

to propagate the angular velocity and the attitude. The subscript $k$ denotes a variable corresponding to the $k$ th discrete timestep for a fixed integration step size $h \in \mathbb{R}$, and $F_{k} \in S O(3)$ is the relative attitude between two adjacent integration steps. The nonstandard moment of inertia matrix is given by $J_{d}=\frac{1}{2} \operatorname{tr}[J] I_{3 \times 3}-J \in \mathbb{R}^{3 \times 3}$, and the moment due to gravity is denoted by $M_{k}=m g \rho \times R_{k}^{T} e_{3} \in \mathbb{R}^{3}$. For a given $\left(R_{k}, \Omega_{k}\right)$, (3) is solved to find $F_{k} \in S O(3)$. Then $\left(R_{k+1}, \Omega_{k+1}\right)$ is obtained by (4) and (5). This yields a map $\left(R_{k}, \Omega_{k}\right) \mapsto\left(R_{k+1}, \Omega_{k+1}\right)$ and this process is repeated. The use of the Lie group variational integrator is desirable since it preserves the orthogonal structure of $S O(3)$ without need of local parameterization or constraints.

\section{A. Uncertainty propagation using linearization on $[0, T]$}

In this method, the uncertainty ellipsoid is propagated by updating both the center of the ellipsoid and the the uncertainty matrix $P$. The center of the uncertainty ellipsoid, denoted by $\hat{R}_{k}, \hat{\Omega}_{k}$, is propagated according to the Lie group variational integrator, initialized by the center of the initial uncertainty ellipsoid. We then assume that the uncertainty ellipsoid is sufficiently small that the flow of points within the uncertainty ellipsoid is well-approximated by the linearized flow of the Lie group variational integrator about this center solution, which we denote by

$$
x_{k+1}=A_{k} x_{k}
$$

where $x_{k}=\left[\zeta_{k} ; \delta \Omega_{k}\right] \in \mathbb{R}^{6}$ and the matrix $A_{k} \in \mathbb{R}^{6 \times 6}$ depends on $\hat{R}_{k}, \hat{\Omega}_{k}$. The expression for $A_{k}$ can be found in [7]. Using this linearization, the uncertainty matrix is propagated according to

$$
P_{k+1}=A_{k} P_{k} A_{k}^{T}
$$

with initial condition given by the initial uncertainty matrix. In this way, the uncertainty ellipsoid is propagated and determined at time $T$.

\section{B. Uncertainty propagation using unscented method on $[0, T]$}

In this method, we compute the propagated uncertainty ellipsoid by using the Lie group variational integrator to propagate a small sample of points selected from the initial uncertainty ellipsoid. Following the conventional wisdom, we choose the 12 points corresponding to the intersection of the boundary of the initial uncertainty ellipsoid and its principal axes. This choice is informed by the fact that if the initial uncertainty ellipsoid is propagated by a linear flow, it will remain an ellipsoid, and it will coincide with the minimal volume ellipsoid containing the the 12 intersection points propagated by the same linear flow.

More explicitly, suppose that the initial uncertainty ellipsoid is given by

$$
\mathcal{E}\left(R_{0}, \Omega_{0}, P_{0}\right)
$$

Let $\lambda^{i} \in \mathbb{R}$ and $\phi^{i}=\left[\phi_{R}^{i} ; \phi_{\Omega}^{i}\right] \in \mathbb{R}^{6}$ be the $i$-th eigenvalue and eigenvector, respectively, of the uncertainty matrix $P_{0} \in \mathbb{R}^{6 \times 6}$ for $i \in\{1,2 \ldots, 6\}$. The intersection of the corresponding principal axis and the ellipsoid boundary is $\sqrt{\lambda_{i}} \phi_{i}$. Then, the 12 intersection points are given by,

$$
\left\{\left(R_{0} \exp \left( \pm \sqrt{\lambda^{i}} \hat{\phi}_{R}^{i}\right), \Omega_{0} \pm \sqrt{\lambda^{i}} \phi_{\Omega}^{i}\right)\right\} \quad i \in\{1,2, \ldots, 6\} .
$$

We propagate these 12 initial conditions using the Lie group variational integrator to determine the 12 values of the attitude and angular velocity at time $T$. We then construct a minimal volume ellipsoid that contains all 12 values of the attitude and angular velocity at time $T$ [8]. From this minimal volume ellipsoid, the center of the ellipsoid, and the uncertainty matrix at time $T$ can be computed. 


\section{Uncertainty propagation using unscented method with re- sampling on $[0, T]$}

The unscented method propagates the same sampled points obtained from the initial uncertainty ellipsoid throughout the entire time period $[0, T]$. In this modification, we partition the time period $[0, T]$ into subintervals and we use the Lie group variational integrator to propagate the 12 sample points throughout each subinterval. At the end of each subinterval, we construct a minimal volume ellipsoid that contains all 12 values of the attitude and angular velocity at the end of that subinterval. We then select a new set of sample points located on the principal axes of this new uncertainty ellipsoid. On the first subinterval, the 12 initial values of the attitude and angular velocity are obtained from the initial uncertainty ellipsoid. In contrast to the previous method which initializes the sample points at the start of the subsequent subinterval by taking the propagated points from the end of the prior subinterval, the current method obtains sample points by resampling from the propagated uncertainty ellipsoid.

If the flow is linear, the re-sampling technique will yield the same estimates as the usual unscented method. If, however, the flow is nonlinear, the re-sampling technique will tend to yield a more conservative estimate, since it captures the deformation of the ellipsoid in the nonlinear flow more explicitly. Indeed, the difference between the two estimates gives an indication of how nonlinear the flow is.

\section{NUMERICAL EXAMPLES}

We apply these methods to the attitude dynamics of the 3D pendulum. The pendulum body is chosen as an elliptic cylinder, and its properties are given by

$$
J=\operatorname{diag}[0.13,0.28,0.17] \mathrm{kg} \cdot \mathrm{m}^{2}, m=1 \mathrm{~kg}, \rho=0.3 e_{3} \mathrm{~m} .
$$

We consider two initial conditions; the first initial condition results in a near-oscillatory attitude flow while the second initial condition results in a highly irregular attitude flow.

Oscillatory attitude flow: The initial uncertainty ellipsoid is characterized by its initial center attitude and angular velocity and initial uncertainty matrix, which are given by

$$
\begin{gathered}
R_{0}=I_{3 \times 3}, \quad \Omega_{0}=[3.0,0.1,0.1] \mathrm{rad} / \mathrm{s}, \\
P_{0}=\operatorname{diag}\left[\left(5 \frac{\pi}{180}\right)^{2}[1,1,1], 0.01^{2}[1,1,1]\right] .
\end{gathered}
$$

It is convenient for visualization to plot the flow of the reduced attitude $R^{T} e_{3}$ on the two-sphere $\mathbb{S}^{2}$. This is because the 3D pendulum has an $\mathbb{S}^{1}$ symmetry given by rotations about the vertical axis, which allows the configuration space to be reduced to the quotient space $S O(3) / \mathbb{S}^{1} \simeq \mathbb{S}^{2}$ [9]. The reduced attitude denotes the direction of gravity in the bodyfixed frame. Plots of the reduced attitude and the angular velocity responses, corresponding to the initial conditions, are shown in Fig. 1 for a time period of 10 seconds.

We apply the three methods to propagate the initial uncertainty through this oscillatory attitude flow for 10 seconds. The integration step size is $h=0.005$, corresponding to 2000 time steps over the length of the simulation. For the unscented method, we find the minimal volume covering

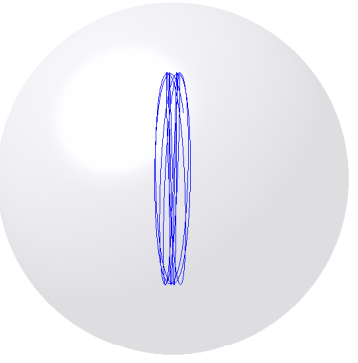

(a) Reduced attitude $\Gamma$

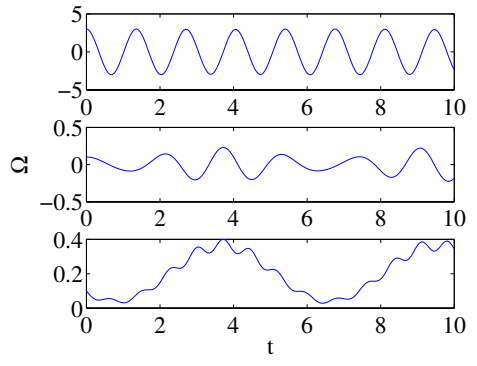

(b) Angular velocity $\Omega$
Fig. 1. Reduced attitude and angular velocity for oscillatory attitude flow (The center of the sphere is the hanging equilibrium where $R^{T} e_{3}=e_{3}$.)

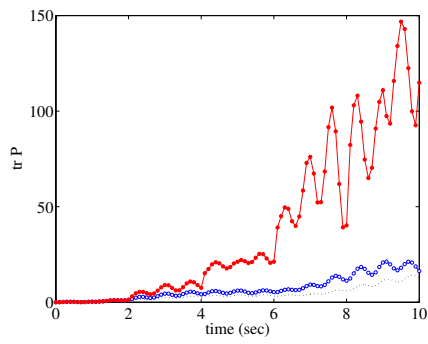

(a) Magnitude of uncertainty matrix

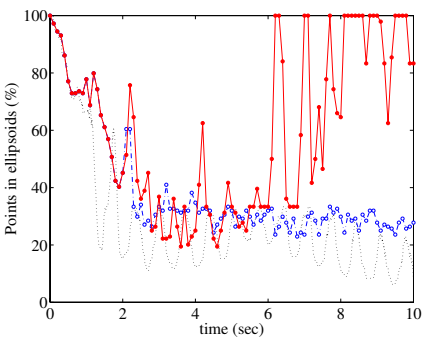
tained in the estimated uncertainty $(\%)$ (b) Percentage of sample points con-

Fig. 2. Uncertainty propagation through oscillatory attitude flow (Linearization: dotted, Unscented: blue, Unscented with re-sampling: red)

ellipsoid every 0.1 seconds. For the unscented method with re-sampling, we find the minimal volume covering ellipsoid every 0.1 seconds, and we choose new set of sample points every 2.0 seconds. To provide a baseline for comparing the performance of each method, we choose sample points in the interior of the initial uncertainty ellipsoid. In particular, we choose 144 points on the level set defined by $x^{T} P_{0}^{-1} x=0.8$, and we numerically integrate each of them.

The magnitudes of the uncertainty matrix and the percentage of sample points (out of 144) contained in the computed uncertainty ellipsoids are shown in Fig. 2. The propagation of uncertainties in the reduced attitude are shown in Fig. 6.

For a short time period, the properties of all methods are similar. The uncertainty ellipsoid computed by the unscented method is larger than for the linearization method, reflecting the nonlinear nature of the dynamics. The re-sampling in the unscented method with re-sampling has the effect of enlarging the uncertainty ellipsoids. Thus, the magnitude of the uncertainty ellipsoid increases rapidly, and the uncertainty ellipsoid from the re-sampling method contains a greater proportion of the sample points after 6 seconds.

The mean precentages of sample points contained in the computed uncertainty ellipsoids are $28.9 \%, 39.8 \%$, and $59.8 \%$, respectively. This suggests that even for this oscillatory attitude flow, the nonlinear effects are so strong that it is difficult to accurately propagate the uncertainty. The computation times are $3.24,45.18$, and 45.84 seconds, respectively on an Intel Pentium M $1.73 \mathrm{GHz}$ processor. 


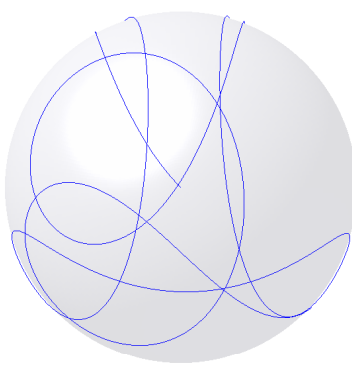

(a) Reduced attitude $\Gamma$

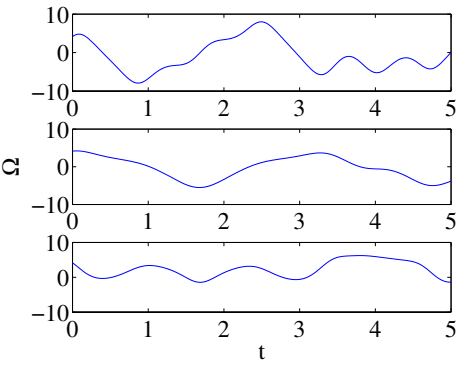

(b) Angular velocity $\Omega$
Fig. 3. Reduced attitude and angular velocity for irregular attitude flow (The center of the sphere is the hanging equilibrium where $R^{T} e_{3}=e_{3}$.)

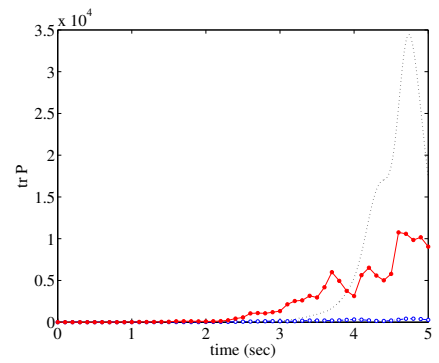

(a) Magnitude of uncertainty matrix

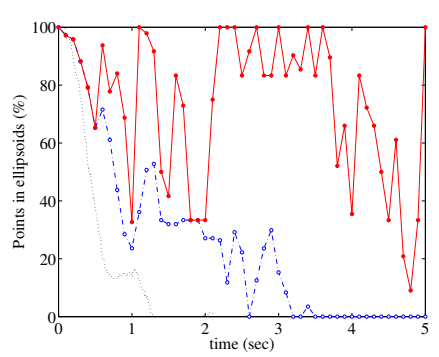

(b) Percentage of sample points contained in the estimated uncertainty $(\%)$
Fig. 4. Uncertainty propagation through irregular attitude flow (Linearization: dotted, Unscented: blue, Unscented with re-sampling: red)

Irregular attitude flow: The initial uncertainty ellipsoid is given by

$$
\begin{aligned}
& R_{0}=I_{3 \times 3}, \quad \Omega_{0}=[4.14,4.14,4.14] \mathrm{rad} / \mathrm{s}, \\
& P_{0}=\operatorname{diag}\left[\left(5 \frac{\pi}{180}\right)^{2}[1,1,1], 0.01^{2}[1,1,1]\right] .
\end{aligned}
$$

The reduced attitude on $\mathbb{S}^{2}$ and the angular velocity responses for 10 seconds corresponding to the center initial conditions are shown in Fig. 3.

We apply the three methods to propagate the initial uncertainty through this irregular attitude flow for 5 seconds. The integration step size is $h=0.002$, corresponding to 2500 time steps over the length of the simulation. For the unscented method, we find the minimal volume covering ellipsoid every 0.1 seconds. For the unscented method with re-sampling, we find the minimal volume covering ellipsoid every 0.1 seconds, and we choose new set of sample points every 0.5 seconds. To compare the properties of each method, we choose 144 initial sample points on the level set of $x^{T} P_{0}^{-1} x=0.8$, and we numerically integrate them.

The magnitudes of the uncertainty matrix and the percentage of sample points (out of 144) contained in the computed uncertainty ellipsoid are shown in Fig. 4. The propagation of uncertainties in the reduced attitude are shown in Fig. 7.

For a short time period, the properties of all methods are similar. The uncertainty ellipsoid computed by the linearization method grows rapidly, but it encloses few points after 1.5 second. The unscented method encloses more points with smaller uncertainty ellipsoids than for the linearization method, but it encloses few points after 3.5 second. The re-sampling in the unscented method with re-sampling has the effect of enlarging the uncertainty ellipsoids. Thus, the size of the uncertainty ellipsoids increase rapidly, and the uncertainty ellipsoid contains more sample points than the other two methods.

The mean numbers of sample points contained in the computed uncertainty ellipsoid are $10.7 \%, 26.3 \%$, and $73.55 \%$, respectively. The computation times are $4.97,43.50$, and 45.56 seconds, respectively on an Intel Pentium M $1.73 \mathrm{GHz}$ processor.

\section{Global Features of the Attitude Flow}

The numerical results presented in the previous section demonstrate the difficulty in obtaining accurate global bounds on attitude solutions that are initialized in an uncertainty ellipsoid. It is claimed that the source of this difficulty is the nonlinear attitude flow of the 3D pendulum, especially the fact that the flow can exhibit chaos and extreme sensitivity to initial conditions. A conceptual description of certain global features of the attitude flow is now provided.

As described in [9], the global dynamics of the 3D pendulum are complicated. There is a 1D hanging equilibrium submanifold of the 3D configuration manifold, consisting of hanging equilibria that differ by a rotation about the vertical. There is also a $1 \mathrm{D}$ inverted equilibrium submanifold consisting of inverted equilibria. Each hanging equilibrium is stable in the sense of Lyapunov. Each inverted equilibrium is unstable, with a 2D stable manifold, a 2D unstable manifold, and a $2 \mathrm{D}$ center manifold. Let $M$ denote the union of all the 2D stable manifolds corresponding to inverted equlibria. This 3D set $M$ plays an important role in understanding the global dynamics of the attitude flow.

Every trajectory in $M$ converges to the inverted equilibrium manifold. Although the set $M$ has measure zero, its existence influences the dynamics of the 3D pendulum attitude flow near $M$. Since $M$ is constructed as the union of the stable manifolds of unstable equilibria, trajectories near $M$ remain near $M$ for an extended period of time. In particular, the closer a trajectory is to $M$ the longer it remains near $M$. In fact, there are trajectories that remain close to $M$ for arbitrarily long time periods. This property is due to the saddle character of each inverted equilibrium.

This can be illustrated by a numerical simulation of the 3D pendulum. Fig. 5 show the attitude deviation from the hanging equilibrium, measured by the quantity $\left\|S^{-1}(\log R)\right\|$, and the angular velocity response for two close initial conditions near the inverted equilibrium; the initial angular velocities are the same, and the initial attitudes only differ by 0.0066 degrees. One initial condition is chosen to be slightly nearer the set $M$. Note that both the attitude and the angular velocity responses have different characteristics; the solution that starts closer to $M$, denoted by dashed lines, remains for more than 2 seconds near the inverted equilibrium, and the angular velocity responses have almost opposite phase. These differences are further increased as the initial condition is chosen to be closer to the set $M$. 

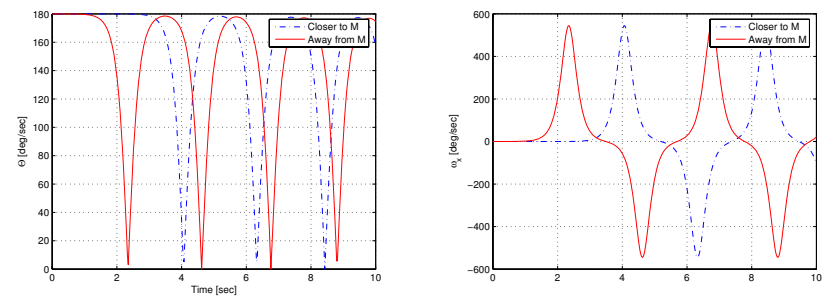

(a) Attitude deviation from the hang- (b) First component of angular velocing equilibrium

ity $\left(\Omega_{1}\right)$

Fig. 5. Effect of a stable manifold of an unstable equilibrium for the 3D pendulum

It should be mentioned that it is difficult to determine exactly the set $M$. One can make use of linear attitude equations near an inverted equilibrium to approximate the tangent space to the stable manifold of that equilibrium. However, this provides only local information about $M$; the non-local properties of the set $M$ are not understood. In practice, to accurately compute the global structure of a stable manifold, one relies on either (i) extremely high-order Taylor approximations of the nonlinear stable manifold for a neighborhood of the equilibrium, which is used to obtain sample points on the stable manifold that are then propagated backwards in time in order to compute the global structure of the stable manifold [10], or (ii) set-oriented techniques based on representing the nonlinear flow map for short times as a Markov chain [11].

This argument demonstrates that the set $M$ has a strong influence on the 3D pendulum dynamics near $M$, with high shearing and thus high sensitivity of the attitude flow near $M$. This is one of the mechanisms leading to the complex nonlinear dynamics of the 3D pendulum and makes it impossible to efficiently compute accurate global bounds on attitude solutions that are initialized in an uncertainty ellipsoid.

\section{CONCLUSIONS}

The Lie group variational integrator is known to provide accurate long-term solutions of the rigid body equations in the presence of an external potential for a given initial attitude and angular velocity; these computed solutions exactly conserve the theoretical conservation properties, namely the symplectic structure, and the angular momentum component about the vertical axis in the case for the 3D pendulum. In addition, it exhibits very good energy behavior, with only a very small bounded energy oscillation, for exponentially long times. Furthermore, the Lie group variational integrator is also known to exactly conserve orthogonality of the computed attitude as a rotation matrix.

It is particularly important to use symplectic methods to propagate individual trajectories in determining uncertainty propagation in Hamiltonian systems since the Gromov nonsqueezing theorem [12] from symplectic geometry places fundamental limits on how the uncertainty of a Hamiltonian mechanical system evolves [13]. Consequently, inaccuracies in the approximate ellipsoidal bounds computed according to the three computational methods introduced do not arise from computational difficulties with the Lie group variational integrator. Rather, the inaccuracies in the approximate ellipsoidal bounds arise from the fact that the attitude flow dynamics are highly nonlinear, with regions wherein the dynamics cannot be adequately approximated by linear dynamics.

With these qualifications, it is clear that the unscented method with resampling is the most accurate of the three proposed methods in propagating the uncertainty. This method can provide a basis for analysis of control and estimation problems for attitude systems such as the 3D pendulum.

For example, attitude estimation operates open loop between measurement times; the analysis in this paper demonstrates the importance of the choice of inter-measurement time in obtaining accurate propagation of the attitude flow between measurement times.

The bottom line demonstrated by the development in this paper is that guaranteed global bounds for attitude dynamics defined by the 3D pendulum, or indeed for any attitude dynamics with a nontrivial potential, are not achievable. That is, there is no universal approach to global and uniform approximation of the attitude flow dynamics.

\section{REFERENCES}

[1] J. Shen, A. Sanyal, N. Chaturvedi, D. Bernstein, and N. McClamroch, "Dynamics and control of a 3D pendulum," Proceedings of the IEEE Conference on Decision and Control, pp. 323-328, 2004.

[2] T. Lee, A. Sanyal, M. Leok, and N. H. McClamroch, "Deterministic global attitude estimation," in Proceedings of the IEEE Conference on Decision and Control, Dec 2006, pp. 3174-3179.

[3] A. Sanyal, T. Lee, M. Leok, and N. H. McClamroch, "Global optimal attitude estimation using uncertainty ellipsoids," Systems and Control Letters, 2007, accepted. [Online]. Available: http: //arxiv.org/abs/math.OC/0606083

[4] M. Kumar, S. Chakravorty, and J. L. Junkins, "A homotopic approach to domain determination and solution refinement for the Fokker-Planck equation," in Proceedings of the American Control Conference, Jul 2007, pp. 1045-1050.

[5] Y. Theodor, U. Shaked, and C. E. de Souza, "A game theory approach to robust discrete-time $H_{\infty}$-estimation," IEEE Transactions on Signal Processing, vol. 42, no. 6, pp. 1486-1495, 1994.

[6] T. Lee, M. Leok, and N. H. McClamroch, "A Lie group variational integrator for the attitude dynamics of a rigid body with application to the 3D pendulum," in Proceedings of the IEEE Conference on Control Application, Aug 2005, pp. 962-967.

[7] - "Attitude maneuvers of a rigid spacecraft in a circular orbit," in Proceedings of the American Control Conference, Jun 2006, pp. 1742-1747.

[8] S. Boyd and L. Vandenberghe, Convex Optimization. Cambridge University Press, 2004.

[9] N. Chaturvedi, T. Lee, M. Leok, and N. H. McClamroch, "Nonlinear dynamics of the 3D pendulum," SIAM Journal on Applied Dynamical Systems, 2007, submitted. [Online]. Available: http://arxiv.org/abs/0707.1196

[10] G. Gómez, W. S. Koon, M. W. Lo, J. E. Marsden, J. Masdemont, and S. D. Ross, "Connecting orbits and invariant manifolds in the spatial restricted three-body problem," Nonlinearity, vol. 17, no. 5, pp. 15711606, 2004.

[11] M. Dellnitz, G. Froyland, and O. Junge, "The algorithms behind GAIO-set oriented numerical methods for dynamical systems," in Ergodic theory, analysis, and efficient simulation of dynamical systems. Berlin: Springer, 2001, pp. 145-174, 805-807.

[12] M. Gromov, "Pseudo holomorphic curves in symplectic manifolds," Invent. Math., vol. 82, no. 2, pp. 307-347, 1985.

[13] F. Hsiao and D. J.Scheeres, "Fundamental constraints on uncertainty evolution in Hamiltonian systems," IEEE Transactions on Automatic Control, vol. 52, no. 4, pp. 686-691, 2007. 
$\therefore$

6

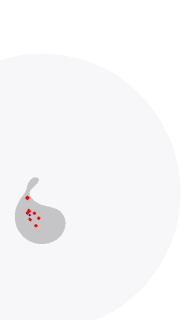

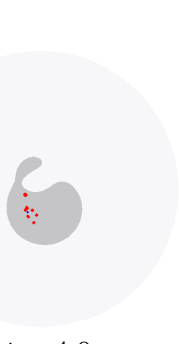

\section{Unscented method}

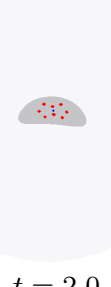

$t=2.0$

$t=4.0$

Unscented with re-sampling
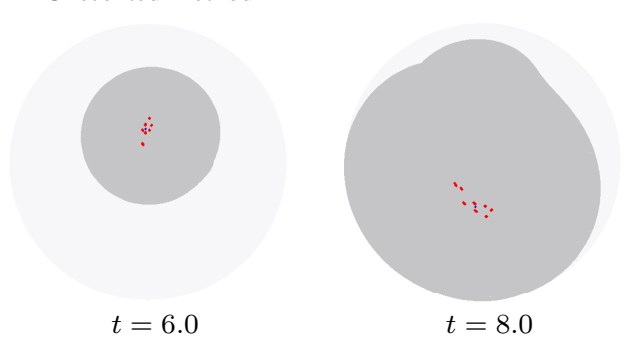

\section{Linearization}

$t=8.0$

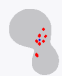

9

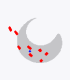

(n)
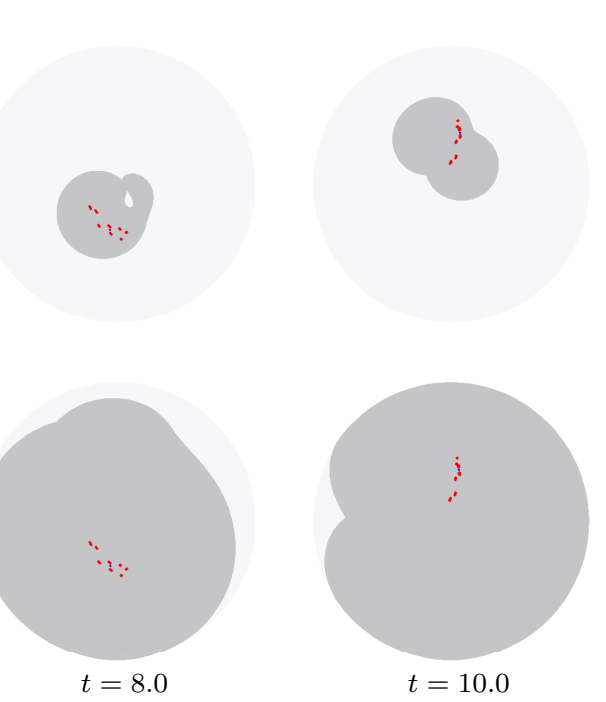

Fig. 6. Uncertainty projected onto the reduced attitude on $\mathbb{S}^{2}$ for oscillatory attitude flow (The center of the sphere is the hanging equilibrium where $\left.R^{T} e_{3}=e_{3}.\right)$

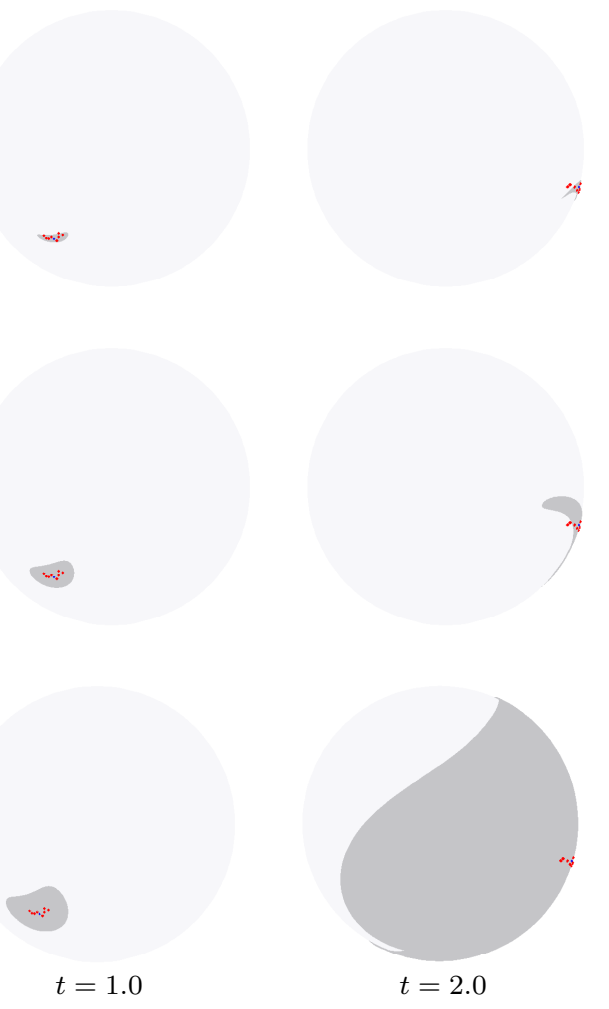

$t=1.0$
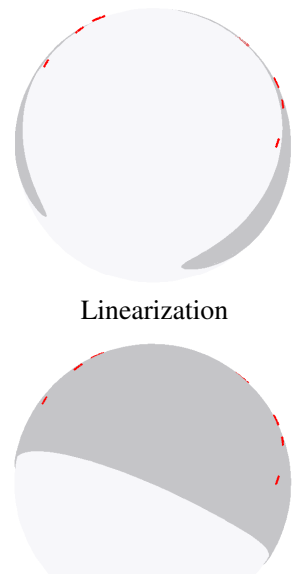

Unscented method

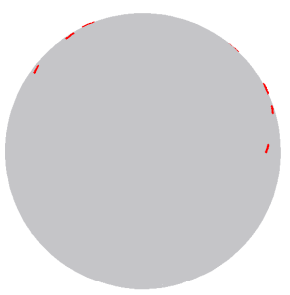

$t=3.0$

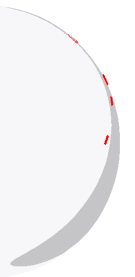

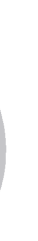
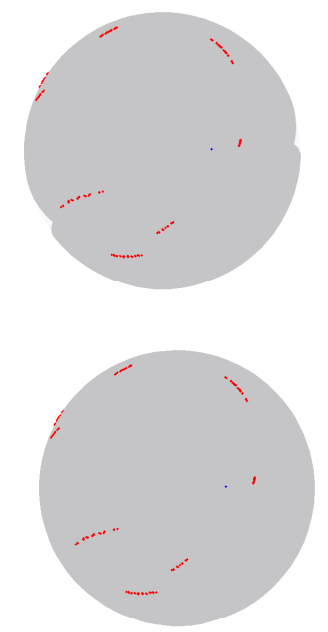

$t=4.0$
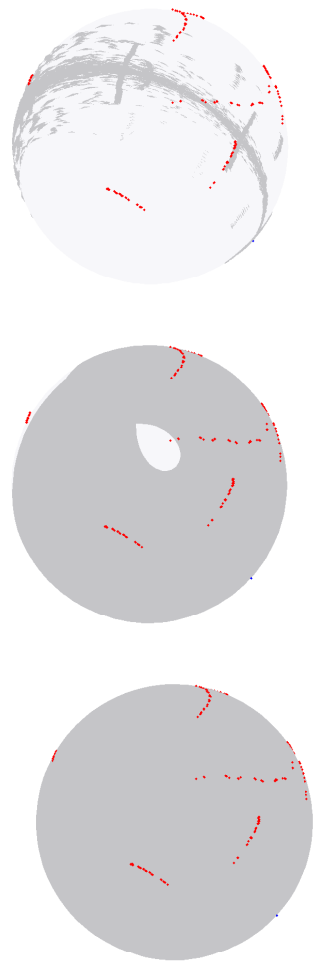

$t=5.0$

Unscented with re-sampling

Fig. 7. Uncertainty projected onto the reduced attitude on $\mathbb{S}^{2}$ for irregular attitude flow (The center of the sphere is the hanging equilibrium where $R^{T} e_{3}=e_{3}$.) 